\title{
Histological appearances of intracranial biopsies obtained using the Cavitron ultrasonic surgical aspirator
}

\author{
RAS BLACKIE, A GORDON* \\ From the Department of Pathology, University of Edinburgh, Edinburgh EH8 $9 A G$, and the \\ ${ }^{*}$ Neuropathology Laboratory, University of Edinburgh, Western General Hospital, Edinburgh EH4 $2 X U$
}

SUMMARY The Cavitron ultrasonic surgical aspirator causes localised ultrasonic tissue fragmentation and has recently been used to remove intracranial tumours. We have examined tissue fragments from 17 operations in which the apparatus was used: in all cases a definitive diagnosis could be made from the material aspirated. This finding has not been previously described.

Prompted by the success of ultrasonic fragmentation and aspiration of cataracts in ophthalmic surgery, the Cavitron ultrasonic surgical aspirator (CUSA) (Cavitron Ultrasonics, Cavitron Corporation, Long Island City, New York) has been recently introduced into neurosurgical practice.'

The equipment has been evaluated in animals in a variety of procedures, ${ }^{23}$ and there are encouraging reports of its use in the treatment of human intracranial and intramedullary tumours. ${ }^{45}$ Because there is no mechanical distortion of adjacent tissue and large blood vessels are undamaged, the CUSA has been used successfully to remove tumours from sites within the central nervous system where other methods have proved difficult or carry an unacceptable risk.

Anticipating that in such circumstances it may be difficult or impossible to obtain a conventional biopsy, we have compared the histology of tissue fragments obtained from the CUSA with the appearances of those obtained by aspiration or excision biopsy.

\section{Patients and methods}

\section{PATIENTS}

Tissue fragments were examined from 17 operations on intracranial tumours in which the CUSA was used in the University of Edinburgh Department of Surgical Neurology, Western General Hospital. In each case a conventional tumour biopsy was

Accepted for publication 4 July 1984 obtained for comparison immediately before the use of the CUSA.

CUSA

The instrument consists of a hollow titanium tube which oscillates longitudinally along its axis at a frequency of $23 \mathrm{kHz}$ with an amplitude of $0 \cdot 1 \mathrm{~mm}$. Tissue within 1 to $2 \mathrm{~mm}$ of its tip is fragmented. The operative field is irrigated with Hartmann's solution at the rate of 3 to $10 \mathrm{ml} / \mathrm{min}$, and the tissue fragments are recovered by a suction device which operates at only 10 to $12 \mathrm{~mm} \mathrm{Hg}$. All the aspirated material is collected into a disposable plastic bottle.

At the end of the operation the plastic bottle was transferred to the laboratory. The top of the bottle was cut off and material floating on the surface of the fluid was discarded; it consisted mainly of air bubbles trapped in fibrin and blood clot. The sediment consisted of isolated, cylindrical pieces of tumour and neural tissue about $1.0 \mathrm{~cm}$ long and 0.2 $\mathrm{cm}$ in diameter. These were washed three times in physiological saline to remove most of the blood and fibrinous debris, and those fragments which settled to the bottom of the container were fixed in $4 \%$

Tumours examined in the 17 patients

\begin{tabular}{ll}
\hline Diagnosis & No of cases \\
\hline Meningioma & 3 \\
Astrocytoma & 6 \\
Oligodendroglioma & 1 \\
Neurilemmoma & 2 \\
Metastatic carcinoma & 4 \\
Myxopapillary ependymoma & 1 \\
\hline
\end{tabular}




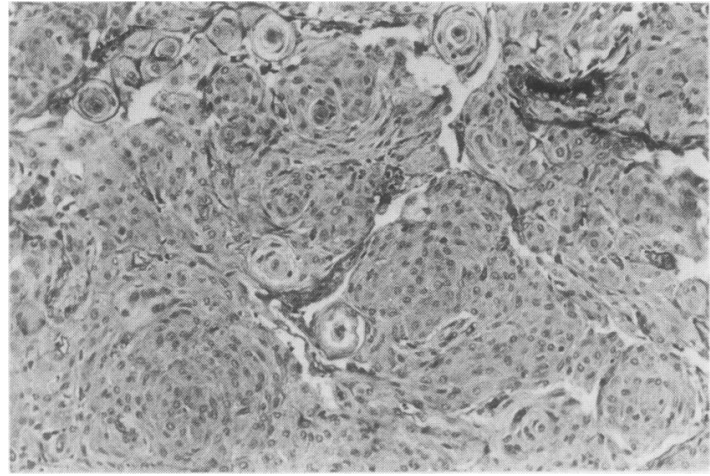

Fig. 1 Meningioma. Haematoxylin and eosin.

Original magnification $\times 160$.

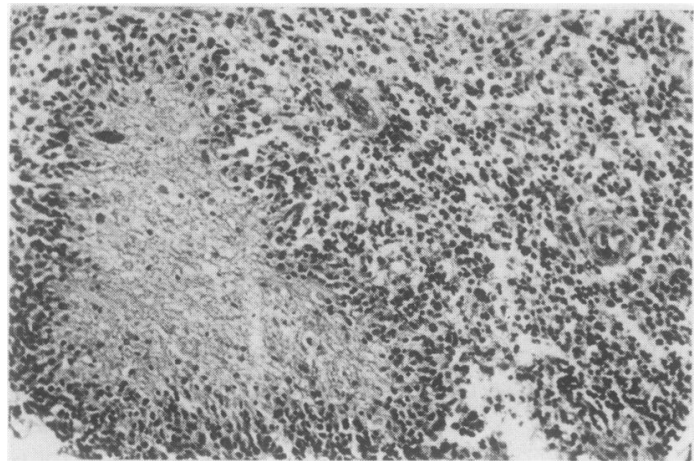

Fig. 2 Perinecrotic pseudopalisading in an astrocytoma.

Haematoxylin and eosin. Original magnification $\times 160$.

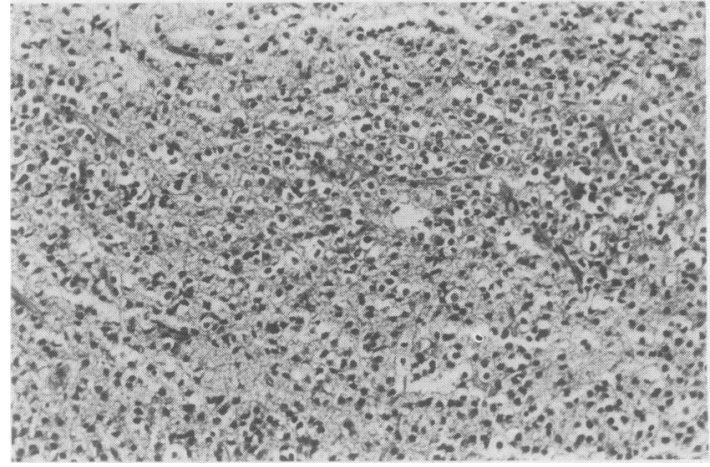

Fig. 3 Oligodendroglioma. Haematoxylin and eosin. Original magnification $\times 160$.

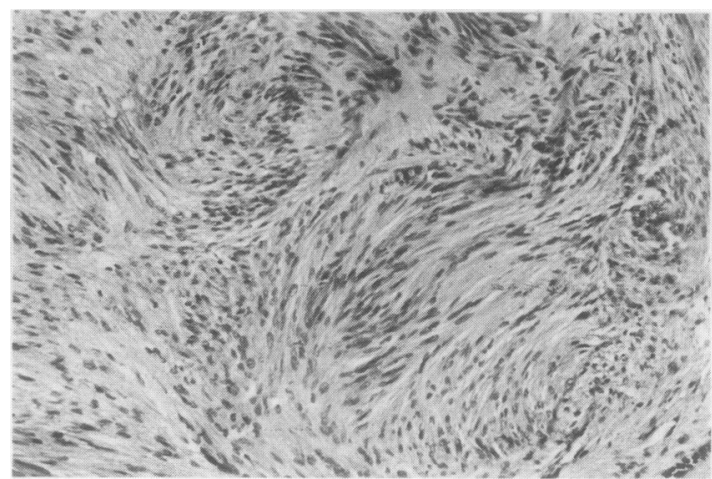

Fig. 4 Neurilemmoma. Haematoxylin and eosin. Original magnification $\times 160$.
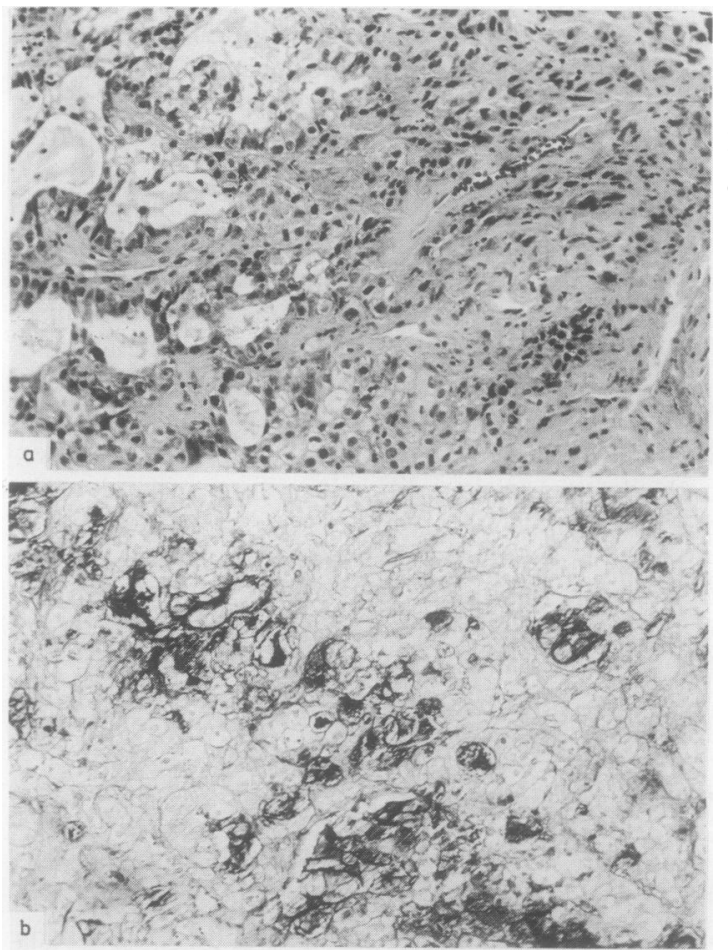

Fig. 5 Metastatic adenocarcinoma. (a) Haematoxylin and eosin. Original magnification $\times 160$.

(b) Alcian blue/periodic acid Schiff. Original magnification $\times 160$. 


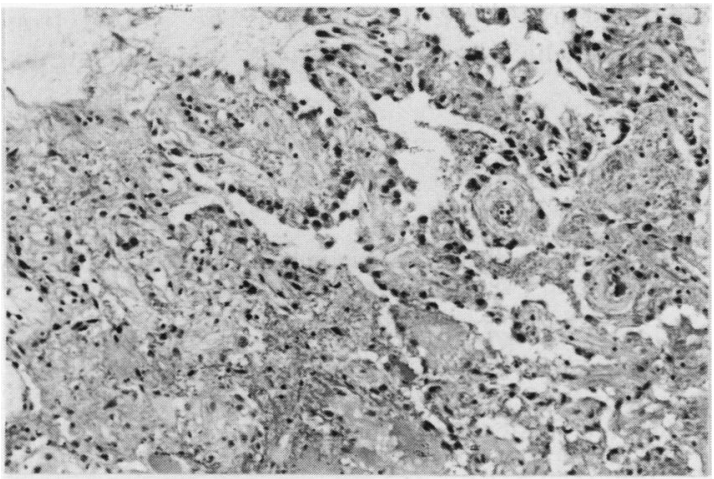

Fig. 6 Myxopapillary ependymoma. Haematoxylin and eosin. Original magnification $\times 160$.

neutral buffered formaldehyde and processed to paraffin wax.

\section{CONVENTIONAL BIOPSIES}

These were fixed and processed in an identical fashion.

\section{STAINING}

Sections from all cases were stained with haematoxylin and eosin and additional sections from selected cases were stained with other commonly used special stains - for example, periodic acid Schiff, phosphotungstic acid haematoxylin, and solochrome cyanin.

\section{Results}

The tumours examined in the 17 patients are listed in the Table.

Examples of the histological appearances of CUSA material are illustrated in Figs. 1-6.

Of the three meningiomas thus treated, two were of the transitional variety and one, attached to the falx, was arachnoidal. The histological appearances of these three tumours were diagnostic; one is illustrated in Fig. 1. The lobulated arrangement of solid masses of tumour cells is clearly seen. The constituent cells have ill defined cell membranes and the nuclei are pale and round or oval.

There were seven gliomas in the series; six of them were astrocytomas of varying grades of malignancy. Five of the astrocytomas were located in a parietal lobe and all showed prominent vascular endothelial proliferation. Perinecrotic pseudopalisading could be identified in two CUSA specimens (Fig. 2). In one CUSA specimen tumour was identified in a Virchow-Robin space, and in another there was pronounced perineuronal infiltration. A feature noted in only one specimen was a degree of artefactual accentuation of microcyst formation.

Although two of the astrocytomas showed areas of oligodendroglial differentiation, there was only one pure oligodendroglioma (Fig. 3). Sections of the CUSA material were diagnostic and the round nuclei and perinuclear haloes characteristic of these tumours were clearly seen. An area containing numerous calcispherules was present in the conventional biopsy specimen, but in the CUSA specimen most of the calcispherules had become separated from the tumoưr mass and were concentrated with the fibrinous debris.

Two VIIIth nerve neurilemmomas were aspirated. The material obtained showed the classic appearances of Antoni type B tissue with compact interlacing bundles of bipolar spindle cells, in which there were areas of nuclear palisading. One example is illustrated in Fig. 4.

Four examples of metastatic carcinoma within the central nervous system were examined. One each was of bronchial, breast, or renal origin, but the primary site of the fourth tumour remains unknown. Stains for mucin were positive in both the CUSA and conventional biopsy specimens. The Alcian blue/periodic acid Schiff stain from the CUSA specimen of the metastatic pulmonary adenocarcinoma is illustrated in Fig. 5(b).

Sections from the biopsies and the CUSA specimens of a myxopapillary ependymoma from the cauda equina showed similar histological features. The tumour had a well defined papillary structure of vascular cores covered by low cuboidal cells with clear cytoplasm (Fig. 6). The prominent vascular component and the recent surgical haemorrhage are evident.

\section{Discussion}

The purpose of this study was to determine whether tumour tissue subjected to ultrasonic fragmentation could be used in diagnostic histopathology. A review of the published work showed that this use of the CUSA fragments has not been previously described. Preliminary discussion with the manufacturers suggested that this matter had not been explored, but they had thought that the "disintegration" would be of such magnitude as to give only cellular fragments.

In all the tumours described a definitive diagnosis could be made using either conventional biopsy material or the material aspirated from the CUSA. The quality of the preparations, however, was in several cases slightly inferior to that obtained by conventional biopsy. This may be due to a combination of factors, among which the most important is 
probably the inevitable mechanical trauma entailed in shaking the tissue loose. Minor histological deterioration was more apparent in the glial tumours: one notable example was the loss of calcispherules from the oligodendroglioma. Other diagnostic features were well preserved, however, and, in the case of microcyst formation, accentuated.

On one occasion the CUSA aspirate was stored overnight at $4^{\circ} \mathrm{C}$ in Hartmann's solution. The quality of preservation and staining was not significantly different from that of material prepared immediately the specimen was received in the laboratory at the end of the operation. This implies a degree of flexibility in the handling procedure, which could, on occasion, be exploited.

Compared with conventional biopsy material, the best preservation was noted with neurilemmomas, meningiomas, and metastatic carcinomas. This finding may reflect the more physically robust nature of these tumours.

Tissue obtained from the CUSA may represent the bulk of the tumour available. In those cases where the initial biopsy is small and has been largely consumed in the preparation of smears or cryostat sections, our preliminary findings suggest that valu- able diagnostic information may be gained from material which would otherwise have been discarded.

\section{References}

' Flamm ES, Ranschoff J, Wuchinich D, Broadwin A. Preliminary experience with ultrasonic aspiration in neurosurgery. Neurosurgery 1978;2:240-5.

2 Derderian GP, Walshaw R, McGehee J. Ultrasonic surgical dissection in the dog spleen. Am J Surg 1982;143:269-73.

${ }^{3}$ Hodgson WJB, Finkelstein JL, Woodriffe P, Jun AHA. Continent anal ileostomy with mucosal proctectomy: a bloodless technique using surgical ultrasonic aspirator in dogs. Br J Surg 1979; 66:857-60.

${ }^{4}$ Epstein N, Epstein F, Allen JC, Aleksic D. Intractable facial pain associated with a ganglioglioma of the cervicomedullary junction: report of a case. Neurosurgical Journal 1982;10:612-6.

${ }^{5}$ Epstein F, Epstein N. Surgical management of holocord intramedullary spinal cord astrocytomas in children: report of three cases. J Neurosurg 1981;54:829-32.

Requests for reprints to: Dr RAS Blackie, Department of Pathology, University Medical School, Teviot Place, Edinburgh EH8 9AG, Scotland. 